\title{
Prognostic factors of patients with mycosis fungoides
}

\author{
Anıl Gülsel Bahalı, Ozlem Su, Fatma Pelin Cengiz, Nazan Emiroğlu, Dilek Bıyık Ozkaya, Nahide Onsun
}

Department of Dermatology, Medical Faculty, Bezmialem Vakif University, Istanbul, Turkey

Adv Dermatol Allergol 2020; XXXVII (5): 796-799

DOI: https://doi.org/10.5114/ada.2020.100491

\begin{abstract}
Introduction: Mycosis fungoides (MF) is the most common type of primary cutaneous T-cell lymphoma. Prognostic factors may help to evaluate the course of the disease and may also be useful in selecting appropriate treatment plans for patients.

Aim: To investigate the potential prognostic factors of MF and their correlations with MF stage.

Material and methods: We evaluated the records of patients with MF who were followed in our lymphoma clinic between 1998 and 2015. Age, sex, disease stage, peripheral blood eosinophilia, eosinophil cationic protein, serum total IgE, lactate dehydrogenase (LDH), and $\beta_{2}$-microglobulin levels were investigated and recorded at the time of diagnosis.

Results: There was a statistically significant positive correlation between high $\beta_{2}$-microglobulin levels and the advanced stage of disease $(p<0.001)$. The older group of patients had statistically significantly higher levels of $\beta_{2}$-microglobulin compared to the younger group $(p=0.001)$. We found strong, significantly positive correlations between disease stage and $\beta_{2}$-microglobulin, LDH, and total IgE levels $(p<0.001$, rho $=0.335 ; p=0.001, r=0.302$; $p=0.001, r=0.311$, respectively). Additionally, there were significantly positive correlations between LDH levels and $\beta_{2}$-microglobulin, total IgE levels ( $p<0.001$, rho $=0.484 ; p=0.001, r=0.212$, respectively). Study limitations: A limited number of patients and the retrospective nature of the study.

Conclusions: We found that $\beta_{2}$-microglobulin was a significant prognostic factor in our study population of MF patients. Also, elevated LDH, $\beta_{2}$-microglobulin, and total IgE levels were correlated with advanced disease. Thus, these parameters can be used together to identify patients who have progressed to the later stages of the disease and who require more aggressive treatment.
\end{abstract}

Key words: mycosis fungoides, prognosis, stage.

\section{Introduction}

Primary cutaneous T-cell lymphomas are a heterogeneous group of extranodal non-Hodgkin's lymphomas that present in the skin. Mycosis fungoides (MF) is the most common type of primary cutaneous T-cell lymphoma. It is characterized by skin infiltration of neoplastic T-lymphocytes. The progression of MF is chronic and slow, and the median age at diagnosis is $55-58$ years. MF may remain stable or may progress to an advanced stage or a leukemic variant. Prognostic factors may help to evaluate the course of the disease and may also be useful in selecting appropriate treatment plans for patients. In addition to the stage, other potential prognostic markers, such as sex, age, increased levels of serum lactate dehydrogenase (LDH), increased $\beta_{2}$-microglobulin, peripheral blood eosinophilia, histological features of folliculotropism (FT), and large-cell transformation have been identified in MF [1-4].

\section{Aim}

The aim of this retrospective study was to investigate the potential prognostic factors of MF and their correlations with MF stage.

\section{Material and methods}

This study's design was a single-centre, retrospective cohort analysis. We evaluated the records of patients with MF who were regularly followed in our lymphoma clinic between 1998 and 2015. Patients with missing re-

Address for correspondence: Anil Gulsel Bahali MD, Department of Dermatology, Medical Faculty, Bezmialem Vakif University, 34093 Fatih, Istanbul, Turkey, phone: +90 2124531700 5912, GSM: +90 505 3893280, fax: +90 212 621 75 80, e-mail: anilirli@yahoo.com Received: 22.08.2019, accepted: 22.10.2019. 
cords were excluded from the study, while 119 patients with MF were included. There was no serious systemic disease which leads to an increase in the level of $\beta_{2}$ microglobulin and LDH in our patients group. The patients were staged according to the TNMB criteria of the National Cancer Institute. Age, sex, disease stage, peripheral blood eosinophilia, eosinophil cationic protein (ECP), serum total IgE count, $\mathrm{LDH}$, and $\beta_{2}$-microglobulin levels were investigated and recorded at the time of diagnosis. We also assessed the significance of these parameters as prognostic factors for the disease process.

\section{Statistical analysis}

Categorical variables were compared by using $\chi^{2}$ tests. Normality tests were used for continuous variables, and continuous variables were compared with the MannWhitney $U$ test and the Student's t-test. The Shapiro Wilk test was used to evaluate the normality. All statistical analyses were performed using the SPSS version 15.0 statistical software program (SPSS, Chicago, IL). Spearman and Pearson correlation analyses were used to find out the correlations between variables according to the normality tests. The results were considered to be statistically significant when the $p$-value was less than 0.05 .

\section{Results}

The study included a total of 119 patients, including 64 (53.8\%) men and 55 (46.2\%) women, for a male-to female ratio of 1.16. The mean age of the patients was $52 \pm 16.3$ years. The overall mean age at diagnosis was $46.3 \pm 15.9$ years (range: $8-86$ years). The stage of disease for all patients at the time of initial diagnosis is shown in Table 1.

The relationships between variables are shown in Table 2. $\beta_{2}$-microglobulin levels increased as the disease stage increased (rho $=0.335, p<0.001$ ). At the time of diagnosis, 98 patients were $<60$ years old and 21 patients were $>60$ years old. The older group of patients had statistically significantly higher levels of $\beta_{2}$-microglobulin compared to the younger group ( $p=$

Table 1. The stage of disease for all patients at the time of initial diagnosis

\begin{tabular}{lc}
\hline Stage & Patients number, $n$ (\%) \\
\hline $1 a$ & $56(47.1)$ \\
\hline $1 b$ & $21(17.6)$ \\
\hline $2 a$ & $21(17.6)$ \\
\hline $2 b$ & $10(8.4)$ \\
\hline $3 a$ & $3(2.5)$ \\
\hline $3 b$ & $3(2.5)$ \\
\hline $4 a$ & $4(3.4)$ \\
\hline $4 b$ & $1(0.8)$ \\
\hline
\end{tabular}

0.001). Also, the mean value and standard deviation was revised as minimum, maximum levels and median level for $\beta_{2}$-microglobulin. The median level of $\beta_{2}$-microglobulin was 2.15, minimum level of $\beta_{2}$-microglobulin was 1.10 and maximum level of $\beta_{2}$-microglobulin was 15.30 .

Of the 119 MF patients, 10 had the folliculotropic variant. The mean level of $\beta_{2}$-microglobulin was 2.6 \pm 1.8 for classical MF and $2.3 \pm 1.1$ for the folliculotropic variant. This difference was not statistically significant $(p=0.520) . \beta_{2}$-microglobulin levels were elevated in 46 (38.6\%) patients, LDH levels were elevated in 16 (13.4\%) patients, and total IgE levels were elevated in 27 (22.6\%) patients.

We found strong, significantly positive correlations between the disease stage and $\beta_{2}$-microglobulin, $L D H$, and total IgE levels $(p<0.001$, rho $=0.335 ; p=0.001$, $r=0.302 ; p=0.001, r=0.311$, respectively). Additionally, there were significantly positive correlations between LDH levels and $\beta_{2}$-microglobulin, total IgE levels $(p<0.001$, rho $=0.484 ; p=0.001, r=0.212$, respectively).

\section{Discussion}

The literature contains many studies that investigated the prognostic factors for MF. Different parameters were evaluated in single or multiple-centre studies in different countries. However, well-defined prognostic parameters for individual risk assessments are rare [1, 2, 5-8]. Large-cell transformation, erythrocyte sedimentation rate, serum interleukin-2 receptor, eosinophilia, clinical response to therapy, histological variables, serum $\mathrm{LDH}$, and $\beta_{2}$-microglobulin have been considered as potential prognostic factors. Some studies reported that these factors were strongly associated with the prognosis of MF, while others reported a limited relationship. Some studies that investigated the superiority of each of these parameters revealed different results [7, 9-11].

In our study, the mean age at the time of diagnosis was younger than that in the literature. Older age has been identified as a prognostic factor in MF. Talpur et al. reported that an age of 66 years or older was an important negative predictive factor. Diamandidou et al. found that an age of 60 years or older was an independent prognostic parameter, similar to our study [1, 10, 11]. Also, in our study there was a correlation between older

Table 2. The relationship between the stage of disease and $\mathrm{LDH}$, total IgE, eosinophil, ECP, $\beta_{2}$-microglobulin ( $\left.\beta 2 \mathrm{M}\right)$

\begin{tabular}{ll}
\hline Stage $-\beta 2 M$ & $p<0.001 ;$ rho $=0.335$ \\
\hline Stage - LDH & $p=0.001 ; r=0.302$ \\
\hline Stage - total lgE & $p=0.001 ; r=0.311$ \\
\hline Stage - eosinophil & $p=0.446 ;$ rho $=-0.070$ \\
\hline Stage - ECP & $p=0.201 ;$ rho $=0.118$ \\
\hline${ }^{*} p<0.05$. &
\end{tabular}


age and serum $\beta_{2}$-microglobulin levels, and both were associated with late-stage MF. Therefore, $\beta_{2}$-microglobulin is viewed as a more meaningful indicator, especially in elderly patients $\beta_{2}$-microglobulin is a $12-k D a$ light chain protein which is associated with tumour mass and growth rate. High levels of serum $\beta_{2}$-microglobulin are associated with a poor prognosis in some malignancies, such as low-grade lymphoma, large-cell lymphoma, and Hodgkin's lymphoma $[12,13]$. The prognostic impact of serum $\beta_{2}$-microglobulin in patients with MF was previously demonstrated in the literature. Talpur et al. evaluated a total of 1,263 patients with MF and Sézary syndrome over a period of 27 years. They suggested that elevated $\beta_{2}$-microglobulin levels were risk factors associated with progression of disease [1]. Diamandidou et al. reported that $\beta_{2}$-microglobulin was a significant prognostic factor and was correlated with a poorer survival. However, they believed that $\beta_{2}$-microglobulin was not an independent prognostic factor because this result was not supported by multivariate analyses [11]. In our study, we found that $\beta_{2}$-microglobulin alone was a significant negative prognostic factor that correlated with the disease stage. It is known that the folliculotropic variant of MF has a worse prognosis than classical MF $[14,15]$. In our study, $\beta_{2}$-microglobulin was not evaluated as a prognostic parameter between these forms.

$\mathrm{LDH}$ is a non-specific marker of tumour burden and several studies have reported that elevated LDH levels are associated with a poor prognosis and that it is a significant prognostic factor for $M F[1,2,6,8,10,11$. Alberti-Violetti et al. studied advanced-stage MF patients and determined that only LDH and advanced age were associated with a poor prognosis [4]. Vonderheid et al. identified soluble IL-2 receptor as a prognostic marker with possibly better specificity than LDH [16]. TancrèdeBohin et al. investigated the prognostic values of blood eosinophilia and LDH in 2004, and reported that blood eosinophilia was a stronger prognostic factor than LDH [17]. The results of our study suggest that elevated $\beta_{2}$-microglobulin is a more significant parameter than LDH, particularly for advanced stages of disease.

In our previous study, which included 78 patients, we found a significant correlation between elevated IgE, ECP, and eosinophil count and advanced stages of MF. We did not evaluate $\beta_{2}$-microglobulin or LDH levels or their associations with the disease [18]. However, in the current study, we investigated all of these and determined that ECP levels and eosinophilia were not related to the disease stage.

\section{Conclusions}

We found that $\beta_{2}$-microglobulin was a significant prognostic factor in our study population of MF patients. In addition, the results suggested that $\beta_{2}$-microglobulin was a more significant negative predictive marker than
LDH. Also, elevated LDH, $\beta_{2}$-microglobulin, and total IgE levels were correlated with advanced disease. Thus, these parameters can be used together to identify patients who have progressed to the later stages of the disease and who require more aggressive treatment. The prognostic parameters have not previously been clearly defined. This may be due to researchers investigating different parameters during different disease stages, and evaluating different populations. The significance of $\beta_{2}$-microglobulin in MF is supported by many studies. However, further prospective studies are needed to evaluate these prognostic factors.

\section{Conflict of interest}

The authors declare no conflict of interest.

\section{References}

1. Talpur R, Singh L, Daulat S, et al. Long-term outcomes of 1,263 patients with mycosis fungoides and Sézary syndrome from 1982 to 2009. Clin Cancer Res 2012; 18: 5051-60.

2. Eklund Y, Aronsson A, Schmidtchen A, Relander T. Mycosis fungoides: a retrospective study of 44 Swedish cases. Acta Derm Venereol 2016; 96: 669-73.

3. Wilcox RA. Cutaneous T-cell lymphoma: 2016 update on diagnosis, risk-stratification, and management. Am J Hematol 2016; 91: 151-65.

4. Alberti-Violetti S, Talpur R, Schlichte M, et al. Advancedstage mycosis fungoides and Sézary syndrome: survival and response to treatment. Clin Lymphoma Myeloma Leuk 2015; 15: 105-12.

5. Desai M, Liu S, Parker S. Clinical characteristics, prognostic factors, and survival of 393 patients with mycosis fungoides and Sézary syndrome in the southeastern United States: a single-institution cohort. J Am Acad Dermatol 2015; 72: 276-85.

6. Suzuki S, Ito K, Ito M, Kawai K. Prognosis of 100 Japanese patients with mycosis fungoides and Sézary syndrome. J Dermatol Sci 2010; 57: 37-43.

7. Kim YH, Liu HL, Mraz-Gernhard S, et al. Long-term outcome of 525 patients with mycosis fungoides and Sezary syndrome: clinical prognostic factors and risk for disease progression. Arch Dermatol 2003; 139: 857-66.

8. Tobisawa S, Honma M, Ishida-yamamoto A, et al. Prognostic factors in 105 Japanese cases of mycosis fungoides and Sézary syndrome: clusterin expression as a novel prognostic factor. J Dermatol Sci 2013; 71: 160-6.

9. Hallermann C, Niermann C, Fischer RJ, Schulze HJ. Erythrocyte sedimentation rate as an independent prognostic factor in mycosis fungoides. Br J Dermatol 2012; 166: 873-4.

10. Scarisbrick JJ, Prince HM, Vermeer MH, et al.; Cutaneous Lymphoma International Consortium Study of Outcome in Advanced Stages of Mycosis Fungoides and Sézary Syndrome. Effect of specific prognostic markers on survival and development of a prognostic model. Clin Oncol 2015; 33: 3766-73.

11. Diamandidou E, Colome M, Fayad L, et al. Prognostic factor analysis in mycosis fungoides/Sézary syndrome. J Am Acad Dermatol 1999; 40: 914-24. 
12. Wang XL, Wang XL, He S, Zhai HL. Association of $\beta_{2}{ }^{-}$ microglobulin with the prognosis of non-Hodgkin's lymphoma: a meta analysis. Int J Clin Exp Med 2015; 8: 3992-9.

13. Tsimberidou AM, Kantarjian HM, Wen S, et al. The prognostic significance of serum B2 microglobulin levels in acute myeloid leukemia and prognostic scores predicting survival: analysis of 1,180 patients. Clin Cancer Res 2008; 14: 721-30.

14. Agar NS, Wedgeworth E, Crichton S, et al. Survival outcomes and prognostic factors in mycosis fungoides/Sézary syndrome: validation of the revised International Society for Cutaneous Lymphomas/European Organisation for Research and Treatment of Cancer staging proposal. J Clin Oncol 2010; 28: 4730-9.

15. Lehman JS, Cook-Norris RH, Weed BR, et al. Folliculotropic mycosis fungoides: single-center study and systematic review. Arch Dermatol 2010; 146: 607-13.

16. Vonderheid EC, Zhang Q, Lessin SR, et al. Use of serum soluble interleukin-2 receptor levels to monitor the progression of cutaneous T-cell lymphoma. J Am Acad Dermatol 1998; 38: 207-20.

17. Tancrède-Bohin E, Ionescu MA, de La Salmonière $P$, et al. Prognostic value of blood eosinophilia in primary cutaneous T-cell lymphomas. Arch Dermatol 2004; 140: 1057-61.

18. Kural YB, Su O, Onsun N, Uras AR. Atopy, IgE and eosinophilic cationic protein concentration, specific IgE positivity, eosinophil count in cutaneous T cell lymphoma. Int I Dermatol 2010; 49: 390-5. 